\title{
Measuring cosmic bulk flows with Type la supernovae from the Nearby Supernova Factory
}

\author{
U. Feindt ${ }^{1}$, M. Kerschhaggl ${ }^{1}$ M. Kowalski ${ }^{1}$, G. Aldering ${ }^{2}$, P. Antilogus ${ }^{3}$, C. Aragon $^{2}$, S. Bailey ${ }^{2}$, C. Baltay ${ }^{4}$, \\ S. Bongard ${ }^{3}$, C. Buton ${ }^{1}$, A. Canto ${ }^{3}$, F. Cellier-Holzem ${ }^{3}$, M. Childress ${ }^{5}$, N. Chotard ${ }^{6}$, Y. Copin ${ }^{6}$, H. K. Fakhouri ${ }^{2,7}$, \\ E. Gangler ${ }^{6}$, J. Guy ${ }^{3}$, A. Kim ${ }^{2}$, P. Nugent ${ }^{8,9}$, J. Nordin ${ }^{2,10}$, K. Paech ${ }^{1}$, R. Pain ${ }^{3}$, E. Pecontal ${ }^{11}$, R. Pereira ${ }^{6}$, \\ S. Perlmutter ${ }^{2,7}$, D. Rabinowitz ${ }^{4}$, M. Rigault ${ }^{6}$, K. Runge ${ }^{2}$, C. Saunders ${ }^{2}$, R. Scalzo ${ }^{5}$, G. Smadja $^{6}$, C. Tao ${ }^{12,13}$, \\ R. C. Thomas ${ }^{8}$, B. A. Weaver ${ }^{14}$, and C. $\mathrm{Wu}^{3,15}$
}

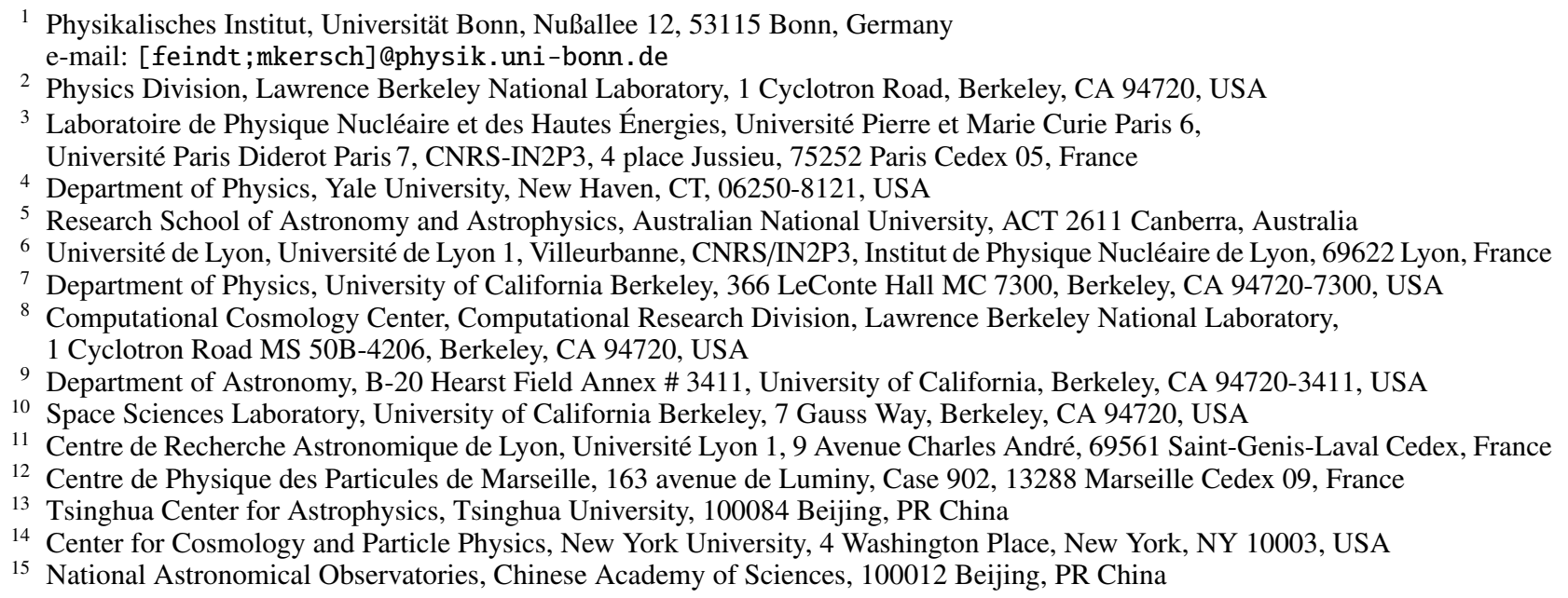

Received 12 May 2013 / Accepted 10 October 2013

\begin{abstract}
Context. Our Local Group of galaxies appears to be moving relative to the cosmic microwave background with the source of the peculiar motion still uncertain. While in the past this has been studied mostly using galaxies as distance indicators, the weight of Type Ia supernovae (SNe Ia) has increased recently with the continuously improving statistics of available low-redshift supernovae. Aims. We measured the bulk flow in the nearby universe $(0.015<z<0.1)$ using $117 \mathrm{SNe}$ Ia observed by the Nearby Supernova Factory, as well as the Union2 compilation of SN Ia data already in the literature.

Methods. The bulk flow velocity was determined from SN data binned in redshift shells by including a coherent motion (dipole) in a cosmological fit. Additionally, a method of spatially smoothing the Hubble residuals was used to verify the results of the dipole fit. To constrain the location and mass of a potential mass concentration (e.g., the Shapley supercluster) responsible for the peculiar motion, we fit a Hubble law modified by adding an additional mass concentration.

Results. The analysis shows a bulk flow that is consistent with the direction of the CMB dipole up to $z \sim 0.06$, thereby doubling the volume over which conventional distance measures are sensitive to a bulk flow. We see no significant turnover behind the center of the Shapley supercluster. A simple attractor model in the proximity of the Shapley supercluster is only marginally consistent with our data, suggesting the need for another, more distant source. In the redshift shell $0.06<z<0.1$, we constrain the bulk flow velocity to $\leq 240 \mathrm{~km} \mathrm{~s}^{-1}(68 \%$ confidence level) for the direction of the CMB dipole, in contradiction to recent claims of the existence of a large-amplitude dark flow.
\end{abstract}

Key words. cosmology: observations - cosmological parameters - large-scale structure of Universe - supernovae: general

\section{Introduction}

The Copernican principle which implies an isotropic Universe on large scales, is one of the major conceptual building blocks of modern cosmology. In this picture, an important task is to explain the apparent motion of our Local Group of galaxies (LG) relative to the cosmic microwave background (CMB) with $v_{\mathrm{LG}}=627 \pm 22 \mathrm{~km} \mathrm{~s}^{-1}$ toward the direction $l=276^{\circ}$ and $b=30^{\circ}$ (Kogut et al. 1993). While the gravitational attraction towards a nearby overdensity is widely accepted as the source of the LG motion, the exact contribution of known overdensities is still under debate since the amplitude of $v_{\mathrm{LG}}$ has not been fully recovered by peculiar velocity measurements in the local universe. On large scales - where the peculiar velocity data become sparser and noisier, thus precluding the reconstruction of a full peculiar velocity field - the mass distribution can be investigated by measuring bulk flows, i.e., coherent motion in large volumes. 
Previous studies of the local bulk flow show possible tension between two sets of results: some analyses have reported possible anomalously large bulk flows on scales of $\sim 100 \mathrm{~h}^{-1} \mathrm{Mpc}$ (Hudson et al. 2004; Watkins et al. 2009; Lavaux et al. 2010; Colin et al. 2011; Macaulay et al. 2012), while others find the bulk flow to be consistent with the expectation from $\Lambda \mathrm{CDM}$ (earliest Courteau et al. 2000; more recently Nusser \& Davis 2011; Nusser et al. 2011; Branchini et al. 2012; Turnbull et al. 2012; Ma \& Scott 2013). Lavaux et al. (2010), for example, found in the $2 \mathrm{MRS}$ galaxy catalog that the LG velocity $v_{\mathrm{LG}}$ and the direction towards the CMB dipole could not be recovered for matter restframes at distances less than $120 h^{-1} \mathrm{Mpc}$. On the other hand, Nusser et al. show for the same catalog and for the $\mathrm{SF}++$ galaxy catalog that the bulk flow amplitude is consistent with $\Lambda$ CDM expectations (Nusser et al. 2011; Nusser \& Davis 2011).

Over larger distances, Kashlinsky et al. (2008) reports a strong and coherent bulk flow out to $d \gtrsim 300 h^{-1}$ Mpc based on measurements of the kinematic Sunyaev-Zeldovich effect of $\mathrm{X}$-ray clusters. According to their latest results (Kashlinsky et al. $2008,2009,2010,2011,2012)$, the bulk flow is $\sim 1000 \mathrm{~km} \mathrm{~s}^{-1}$ in the direction of the $\mathrm{CMB}$ dipole up to a distance of at least $\sim 800 \mathrm{Mpc}$. The large-scale structure formation predicted by the $\Lambda \mathrm{CDM}$ model does not explain such high values for bulk flows at these distances. A possible explanation for this "dark flow" is a tilt imprinted on our horizon by pre-inflationary inhomogeneities (Grishchuk \& Zeldovich 1978; Turner 1991). However, this claim has been questioned in various studies (Keisler 2009; Osborne et al. 2011; Mody \& Hajian 2012; Lavaux et al. 2013) and recently possibly rejected by the Planck Collaboration (2013) ${ }^{1}$.

At shorter distances, the mass distribution can be studied more precisely by reconstructing the peculiar velocity field using large galaxy catalogs (Erdoğdu et al. 2006; Lavaux et al. 2010). The contribution of overdensities on those scales to the LG motion has long been studied. Lynden-Bell et al. (1988) measured a flow that suggested a great attractor (GA) at about $43 h^{-1} \mathrm{Mpc}$. However, Kocevski \& Ebeling (2006) found that the GA only accounts for $44 \%$ of the dipole anisotropy in a large X-ray cluster sample, with the rest evidently caused by more distant sources such as the Shapley supercluster (SSC) at a distance of $105-165 h^{-1} \mathrm{Mpc}(0.035<z<0.055)$ in the direction $l=306.44^{\circ}, b=29.71^{\circ}$ (Shapley 1930; Scaramella et al. 1989; Raychaudhury et al. 1991). In addition to attraction due to nearby overdensities, outflows from local voids contribute to the LG motion. Tully et al. (2008) decomposed the peculiar motion of the Local Sheet in which the LG is embedded into three almost orthogonal components. Two of these are attributed to attraction by the Virgo and Centaurus clusters, while the other is a velocity of $259 \mathrm{~km} \mathrm{~s}^{-1}$ toward $l=209.7^{\circ}, b=-2.6^{\circ}$, away from the Local Void. Owing to the increasing sparseness of data on larger scales, it is still not clear whether convergence of $v_{\mathrm{LG}}$ can be achieved including the SSC or if a gravitational source extending even further out is responsible for the CMB dipole (Lavaux et al. 2010). Studying bulk flows at large distances will yield valuable information on the source of the LG motion.

When determining the velocity of the LG, other effects that contribute to the CMB dipole need to be considered as well. Wiltshire et al. (2013) analyzed the Hubble flow variance of

\footnotetext{
1 However, Atrio-Barandela (2013) argues that the uncertainties were overestimated in this study, and therefore the bulk flow measured by Planck is more significant than reported and is consistent with the previous results of Kashlinsky et al. (2010).
}

4534 galaxy distances and find that the mean of the Hubble parameter of consecutive redshift shells is more compatible with its global value when using the $\mathrm{LG}$ rather than the CMB as rest frame. In addition to a local boost, it is suggested that the CMB dipole could be attributed to differences in the apparent distance to the surface of last scattering mediated by foreground structures on the $60 \mathrm{~h}^{-1} \mathrm{Mpc}$ scale.

Peculiar velocity fields in the nearby Universe have long been investigated using galaxies as distance indicators, where the distance is estimated through the Tully-Fisher relation (Tully \& Fisher 1977), the fundamental plane (Djorgovski \& Davis 1987; Dressler et al. 1987) or surface brightness fluctuations (Tonry \& Schneider 1988), see, e.g., Watkins et al. (2009) and Lavaux et al. (2010) and references therein. However, galaxies as distance indicators generally range out around $100 h^{-1} \mathrm{Mpc}$ as the accuracy per galaxy becomes low and the observational cost large. Supernovae of Type Ia (SNe Ia), on the other hand, are bright standardizable candles, i.e., showing a brightness dispersion of $\sim 10 \%$ after empirical corrections (Phillips 1993), and thus are alternative tracers of bulk flow motions exceeding by far the redshift range of galaxy distance indicators. While brightness and standardizability favor SNe Ia, the lack of large samples has limited their use to only a few studies so far. Early studies of nearby $\mathrm{SN}$ data showed that the motion of the LG is consistent with the measured dipole seen in the CMB (Riess et al. 1995), and this has been confirmed a number of times since then (Haugbølle et al. 2007; Gordon et al. 2007, 2008; Weyant et al. 2011). One of the most recent studies of a dataset of 245 nearby SNe Ia resulted in a bulk flow towards $l=319^{\circ} \pm 18^{\circ}$, $b=7^{\circ} \pm 14^{\circ}$ at a rate of $249 \pm 76 \mathrm{~km} \mathrm{~s}^{-1}$ (Turnbull et al. 2012). Moreover, Schwarz \& Weinhorst (2007) discovered a statistically significant hemispheric anisotropy at $>95 \%$ confidence level in several SN Ia datasets, for SNe at $z<0.2$. They found an asymmetry between the north and south equatorial hemispheres. Kalus et al. (2013) recently verified with a larger dataset that the asymmetry does not contradict $\Lambda \mathrm{CDM}$ expectations.

Since the coordinates for the GA, SSC and CMB dipole are compatible across the existing studies, a clarification of the situation described above can be approached through the study of redshift shells. Recently Colin et al. (2011) investigated anisotropies in discrete redshift shells using the Union2 compilation of Type Ia SNe (Amanullah et al. 2010). This z-tomography apparently revealed a SSC signature imprinted on the nearby Hubble flow. However, this existing SN Ia sample lacks sufficient variance-weighted depth. Therefore new data are needed to clarify the nature of nearby bulk flows.

In this paper we present an anisotropy study using new SN distance measurements from the Nearby Supernova Factory (SNFACTORY, Aldering et al. 2002), a project that focuses on a large set of spectrophotometrically observed SNe Ia in the redshift range $0.03<z<0.08\left(90-240 h^{-1} \mathrm{Mpc}\right)$. The SNFactory dataset used here consists of $117 \mathrm{SNe}$ Ia and more than doubles the number of distance measurements in this redshift range and provides - for the first time with conventional distance measurements - sensitivity to a bulk flow on distance scales exceeding that of the SSC. Our starting point for the analysis were the findings of Colin et al. (2011) who report a tentative observation of a backside infall behind the SSC. We also investigate the dark flow reported by Kashlinsky et al. (2010).

The paper is organized as follows: the SNFActory dataset is presented in Sect. 2, along with the procedure used to combine it with the Union2 SN compilation. In Sect. 3 the methods used to analyze bulk flows are described. The data are divided into redshift shells and fit for a bulk velocity (the dipole fit (DF) method 
based on Bonvin et al. 2006). An alternative approach to test for anisotropies, the smoothed residuals (SR) method (Colin et al. 2011) is employed on the data as well. The results of this study are presented in Sect. 4. An analysis of a simplified model of a SSC-like overdensity is presented in Sect. 5. Finally, a discussion of the findings is given in Sect. 6 .

\section{Datasets}

The datasets used in this study are a subset of 117 SNe Ia from the Nearby Supernova Factory (Aldering et al. 2002) dataset described below, and the Union2 compilation of $557 \mathrm{SNe}$ Ia (Amanullah et al. 2010) as well as the combination of the two. In order to study the redshift dependence of a possible anisotropy, the data were divided in redshift shells, following Colin et al. (2011). The available number of distances per redshift bin is summarized in Table 1.

\subsection{Nearby Supernova Factory data}

The SNFactory is devoted to the study of $\mathrm{SNe}$ Ia in the nearby Hubble flow $(0.03<z<0.08)$ for use in cosmological analyses. It features a custom-built two-channel Supernova Integral Field Spectrograph (SNIFS) mounted on the University of Hawaii $2.2 \mathrm{~m}$ telescope at Mauna Kea. SNIFS has a field of view of $6^{\prime \prime} \times 6^{\prime \prime}$. It produces a spectrum for each element of a $15 \times 15$ microlens array, resulting in a $3 \mathrm{D}$ data cube at a given epoch. Each cube contains the full information of the SN signal, host galaxy and sky as a function of wavelength $\lambda$ and sky position $x, y$, resulting in 225 spectra per data cube. The measurement is thus independent of any filter characteristics and $K$-corrections. The SN signal is extracted from the data cube using point spread function fitting techniques, a calibration procedure that fits for nightly atmospheric extinction (Buton et al. 2013) as well as host galaxy subtraction (Bongard et al. 2011). A more complete description of SNFACTORY, SNIFS and its operation can be found in Aldering et al. (2002) while more information on the data processing is presented in Aldering et al. (2006) and updated in Scalzo et al. (2010).

Lightcurves were synthesized from the flux-calibrated spectra as recorded with the SNIFS instrument. For this, box filters were applied roughly matching $B, V$, and $R$ (see Bailey et al. 2009 for details). The SN $B$-band restframe magnitudes at maximum light, $m_{B}$, were then extracted using the SALT2 lightcurve fitting algorithm (Guy et al. 2007). As a result, the distance modulus, $\mu_{B}$, for each SN can be obtained after correcting for an empirical width- and color-luminosity relation (Phillips 1993; Tripp 1998):

$\mu_{B}=m_{B}-M+\alpha \cdot x_{1}-\beta \cdot c$,

where $m_{B}, x_{1}$ and $c$ are determined in the lightcurve fit ${ }^{2}$ while $\alpha$, $\beta$ and the absolute magnitude $M$ are parameters in the fit of the SN Hubble diagram (Guy et al. 2007).

The dataset used in the analyses is obtained from the full SNFACTORY data set available at the start of this study through application of quality criteria:

1. the SALT2 lightcurve fit includes more than 5 independent epochs;

\footnotetext{
2 Here $x_{1}$ is a "stretch factor" parameterizing the SN lightcurve and $c$ describes the difference in color between the observed SN and a template SN after correcting for Galactic extinction (Guy et al. 2007).
}

2. the normalized median absolute deviation (nMAD) of the fit residuals over all used filters is smaller than 0.2 mag;

3. there is less than a $20 \%$ rejection rate of data points in SALT2;

4. removal of suspected super-Chandrasekhar SNe. (Scalzo et al. 2012).

A sample of $117 \mathrm{SNe}$ Ia passed the selection criteria. The lightcurve parameters and Hubble residuals for most of the SNe have been published in previous SNFACTORY papers (Bailey et al. 2009; Chotard et al. 2011). The systemic host redshifts are published in Childress et al. (2013a). The data were then grouped in redshift bins as given in Table 1 . Figure 1 shows the SN distribution on the sky for three different bins for the Union2 and SNfactory datasets respectively. The SNFactory dataset is limited to declinations easily observable from Mauna Kea $\left(-25^{\circ}<\right.$ $\delta<65^{\circ}$ ) and therefore covers approximately $70 \%$ of the sky.

\subsection{Union2 and the combined dataset}

Additionally we analyzed the Union2 compilation (Amanullah et al. 2010) of 577 SNe of which 165 are at redshifts below $z=$ 0.1 (Hamuy et al. 1996; Riess et al. 1998, 1999, 2007; Perlmutter et al. 1999; Knop et al. 2003; Tonry et al. 2003; Barris et al. 2004; Krisciunas et al. 2005; Astier et al. 2006; Jha et al. 2006; Miknaitis et al. 2007; Amanullah et al. 2008; Holtzman et al. 2008; Kowalski et al. 2008; Hicken et al. 2009). However, 109 of these low-redshift SNe are at $z<0.035$ while the SNFActory dataset has a more even distribution of redshifts.

We checked both datasets for SNe that are close to galaxy clusters using the NASA/IPAC Extragalactic Database (NED). As the velocities of such SNe are dominated by virial motion, the redshift of the cluster should be used instead of that of the SN. We found $11 \mathrm{SNe}$ having a projected separation within $1 \mathrm{Mpc}$ of a confirmed cluster and with redshift differences within the velocity dispersion of that cluster.

After analyzing the datasets separately, we combined them to create a new sample of $279 \mathrm{SNe}^{3}$ spanning $0.015<z<0.1$. As the absolute magnitudes, $M$, of the SNe and the parameters $\alpha$ and $\beta$ of Eq. (1) were determined separately for the datasets following the Union procedure, their normalizations may differ. This would lead to a larger than usual scatter of the Hubble residuals. Therefore we determined an offset between the distance moduli in a $\chi^{2}$ fit of a flat $\Lambda$ CDM cosmology, with $\Omega_{M}$ and the absolute corrected magnitude $M$ of SNe the parameters of the fit. These parameters were determined using all $693 \mathrm{SNe}$ of both datasets and were left blinded to preserve the impartiality of ongoing SNFACTORY cosmology analyses.

\section{Analysis}

For the study of anisotropies and bulk flows present in SN Ia data two methodologically distinct techniques were chosen. A dipole fit (DF) method based on Bonvin et al. (2006) is used to determine the bulk flow velocity in redshift shells. As a cross-check we reimplement the smoothed residuals (SR) method previously used by Colin et al. (2011). The significance of both methods is determined by randomly resampling the directions of the SNe.

3 SN 2005eu (Hicken et al. 2009) was also observed by the SNFactory as SNF20051003-004. We included it in both data sets when analyzing them separately. Since the results agree we only used the Union2 value in the combined dataset. 
Table 1. Reconstructed directions (in Galactic coordinates) and $p$-values of maximum anisotropy according to a dipole fit and the methods of smoothed residuals in different redshift bins for the Union2 and SNFACTORY datasets and their combination.

\begin{tabular}{|c|c|c|c|c|c|c|c|c|c|c|c|c|}
\hline \multicolumn{3}{|l|}{ Union2 } & \multicolumn{4}{|c|}{ Dipole fit } & \multicolumn{3}{|c|}{ Smoothed residuals } & \multicolumn{3}{|c|}{ Sample characteristics } \\
\hline & $N_{\mathrm{SN}}$ & $z$ & $l$ & $b$ & $v_{\mathrm{DF}}\left[\mathrm{km} \mathrm{s}^{-1}\right]$ & $p$-value & $l$ & $b$ & $p$-value & $|\overline{\boldsymbol{n}}|$ & $l$ & $b$ \\
\hline & 109 & $0.015-0.035$ & $290(22)$ & $15(18)$ & 292(96) & 0.004 & 300 & 30 & 0.030 & 0.287 & 144 & 54 \\
\hline & 16 & $0.035-0.045$ & $331(59)$ & $-7(37)$ & $496(468)$ & 0.316 & 310 & 20 & 0.230 & 0.200 & 148 & 17 \\
\hline & 17 & $0.045-0.060$ & $39(45)$ & $-36(28)$ & $870(490)$ & 0.068 & 60 & -30 & 0.234 & 0.327 & 243 & -58 \\
\hline & 23 & $0.060-0.100$ & $54(93)$ & $-10(53)$ & $509(601)$ & 0.465 & 70 & -30 & 0.724 & 0.340 & 139 & -70 \\
\hline & 55 & $0.100-0.200$ & $256(90)$ & $-18(34)$ & $1238(1975)$ & 0.135 & 240 & 40 & 0.524 & 0.666 & 120 & -64 \\
\hline & 62 & $0.200-0.300$ & $14(131)$ & $11(75)$ & $1221(1457)$ & 0.327 & 0 & 60 & 0.644 & 0.805 & 91 & -57 \\
\hline & 62 & $0.300-0.400$ & $257(84)$ & $-36(27)$ & $2590(2841)$ & 0.102 & 130 & -80 & 0.184 & 0.581 & 131 & -56 \\
\hline & 58 & $0.400-0.500$ & $161(48)$ & $28(29)$ & $4190(4014)$ & 0.208 & 220 & 60 & 0.337 & 0.211 & 166 & 12 \\
\hline & 44 & $0.500-0.600$ & $15(100)$ & $-17(33)$ & 3977(4113) & 0.307 & 20 & 10 & 0.464 & 0.114 & 167 & -42 \\
\hline & 50 & $0.600-0.800$ & $343(81)$ & $-50(43)$ & $5576(4279)$ & 0.107 & 30 & -70 & 0.072 & 0.137 & 127 & -7 \\
\hline & 60 & $0.800-1.400$ & $75(55)$ & $-14(28)$ & $7238(8933)$ & 0.538 & 280 & -60 & 0.949 & 0.364 & 131 & 37 \\
\hline \multicolumn{13}{|c|}{ SNFACTORY } \\
\hline & 20 & $0.015-0.035$ & $104(95)$ & $26(44)$ & $229(410)$ & 0.806 & 330 & 50 & 0.885 & 0.409 & 92 & -25 \\
\hline & 20 & $0.035-0.045$ & $286(70)$ & $-7(42)$ & $484(516)$ & 0.313 & 270 & 20 & 0.590 & 0.311 & 82 & -15 \\
\hline & 21 & $0.045-0.060$ & $330(48)$ & $48(46)$ & $941(390)$ & 0.006 & 310 & 60 & 0.004 & 0.497 & 79 & -43 \\
\hline & 54 & $0.060-0.100$ & $252(134)$ & $5(75)$ & $232(360)$ & 0.791 & 270 & 30 & 0.941 & 0.394 & 85 & -12 \\
\hline \multicolumn{13}{|c|}{ Union2+SNFACTORY } \\
\hline & $128^{*}$ & $0.015-0.035$ & $298(25)$ & $15(20)$ & 243(88) & 0.010 & 300 & 30 & 0.074 & 0.252 & 130 & 45 \\
\hline & 36 & $0.035-0.045$ & $302(48)$ & $-12(26)$ & $452(314)$ & 0.131 & 300 & 20 & 0.186 & 0.211 & 105 & -4 \\
\hline & 38 & $0.045-0.060$ & $359(32)$ & $14(27)$ & $650(398)$ & 0.075 & 340 & 60 & 0.132 & 0.340 & 87 & -67 \\
\hline & 77 & $0.060-0.100$ & $285(234)$ & $-23(112)$ & $105(401)$ & 0.885 & 0 & -90 & 0.999 & 0.324 & 91 & -30 \\
\hline
\end{tabular}

Notes. The rightmost column shows the weighted mean direction of SNe, as described in Sect. 4.3. * SN 2005eu (= SNF20051003-004) is included in both datasets. The Union 2 measurement was used for the combined datasets.

\subsection{Peculiar velocity dipole}

A bulk flow toward a certain direction will be observable in the supernova data as a dipole in the peculiar velocity field. In order to quantify the impact of a bulk flow imprinted in the velocity field of SN Ia data, luminosity distances $d_{\mathrm{L}}\left(z, v_{\mathrm{DF}}\right)$ are fit with a simple dipole model. Following Bonvin et al. (2006)

$d_{\mathrm{L}}\left(z, v_{\mathrm{DF}}, \theta\right)=d_{\mathrm{L}}^{(0)}(z)+d_{\mathrm{L}}^{(\mathrm{dipole})}\left(z, v_{\mathrm{DF}}, \theta\right)$

where

$d_{\mathrm{L}}^{(0)}(z)=c(1+z) \int_{0}^{z} \frac{\mathrm{d} z^{\prime}}{H\left(z^{\prime}\right)}$

$z$ is the cosmological redshift, $v_{\mathrm{DF}}$ is the dipole velocity amplitude, $\theta$ is the angle between the line of sight w.r.t. a single $\mathrm{SN}$ and the dipole direction and $H(z)$ represents the Hubble parameter.

The dipole term $d_{\mathrm{L}}^{\text {(dipole) }}\left(z, v_{\mathrm{DF}}, \theta\right)$ can be written as

$d_{\mathrm{L}}^{(\mathrm{dipole})}\left(z, v_{\mathrm{DF}}, \theta\right)=\frac{v_{\mathrm{DF}}(1+z)^{2}}{H(z)} \cdot \cos (\theta)$.

Thus the bulk flow can be determined by comparing the measured distance moduli, $\mu_{i}$, and measured redshifts, $z_{i}$, to the prediction from the $\Lambda \mathrm{CDM}$ Hubble law, i.e., by minimizing the expression

$\chi^{2}=\sum_{i} \frac{\left|\mu_{i}-5 \log _{10}\left(\left(d_{\mathrm{L}}^{(0)}\left(z_{i}\right)-d_{\mathrm{L}}^{(\text {dipole })}\left(z_{i}, v_{\mathrm{DF}}, \theta_{i}\right)\right) / 10 \mathrm{pc}\right)\right|^{2}}{\sigma_{\mathrm{B}}^{2}}$ with the bulk flow velocity $v_{\mathrm{DF}}$ and its direction as parameters. The uncertainties, $\sigma_{i}$, include a sample-independent intrinsic scatter of $\sim 0.15$. Note that because $d_{\mathrm{L}}^{\text {(dipole) }}$ is linear in $v_{\mathrm{DF}}$, negative values of $v_{\mathrm{DF}}$ are permitted mathematically. Fitting $\chi^{2}$ in opposite directions would result in a sign change of the velocity. To break this degeneracy we forced $v_{\mathrm{DF}}$ to be non-negative. Therefore the resulting $\chi^{2}$ value is equal to the value for the $\Lambda C D M$ Hubble law for all directions on one half of the sky. The uncertainties $(68 \% \mathrm{CL})$ of $v_{\mathrm{DF}}$ and its direction are determined from minimizing $\chi^{2}$, and finding the parameter values for which $\chi^{2}$ is increased by $\Delta \chi^{2}=1$. To compute the significance of a small $\chi^{2}$, the SN Ia data are resampled 5000 times, i.e., taking the same $\mu_{i}$ but with $\mathrm{SN}$ coordinates $\left(\theta_{i}, \phi_{i}\right)$ randomly interchanged. The fraction of random realizations that have a smaller $\chi^{2}$ than the observed one gives us the $p$-value of the measured bulk flow. In this procedure, we do not account for cosmic variance when determining the $p$-value and may hence slightly underestimate the uncertainties. Assuming cosmic variance terms of $\sim 100 \mathrm{~km} \mathrm{~s}^{-1}$, we expect the intrinsic dispersion to dominate the error budget for all but the nearest redshift shell.

In Eq. (5) we do not include the correlation in peculiar velocity between nearby $\mathrm{SNe}$ expected due to large-scale structure (Hui \& Greene 2006; Gordon et al. 2007, 2008; Davis et al. 2011), as one goal of the analysis is to establish the large-scale correlation empirically. The bulk flows tested would result in correlations between data points that mostly exceed the ones expected on average due to large-scale structure, thus including both would lead to an overestimation of the covariance. To test this we have compared the covariance expected for a bulk flow of $300 \mathrm{~km} \mathrm{~s}^{-1}$ to that derived from a full power spectrum 

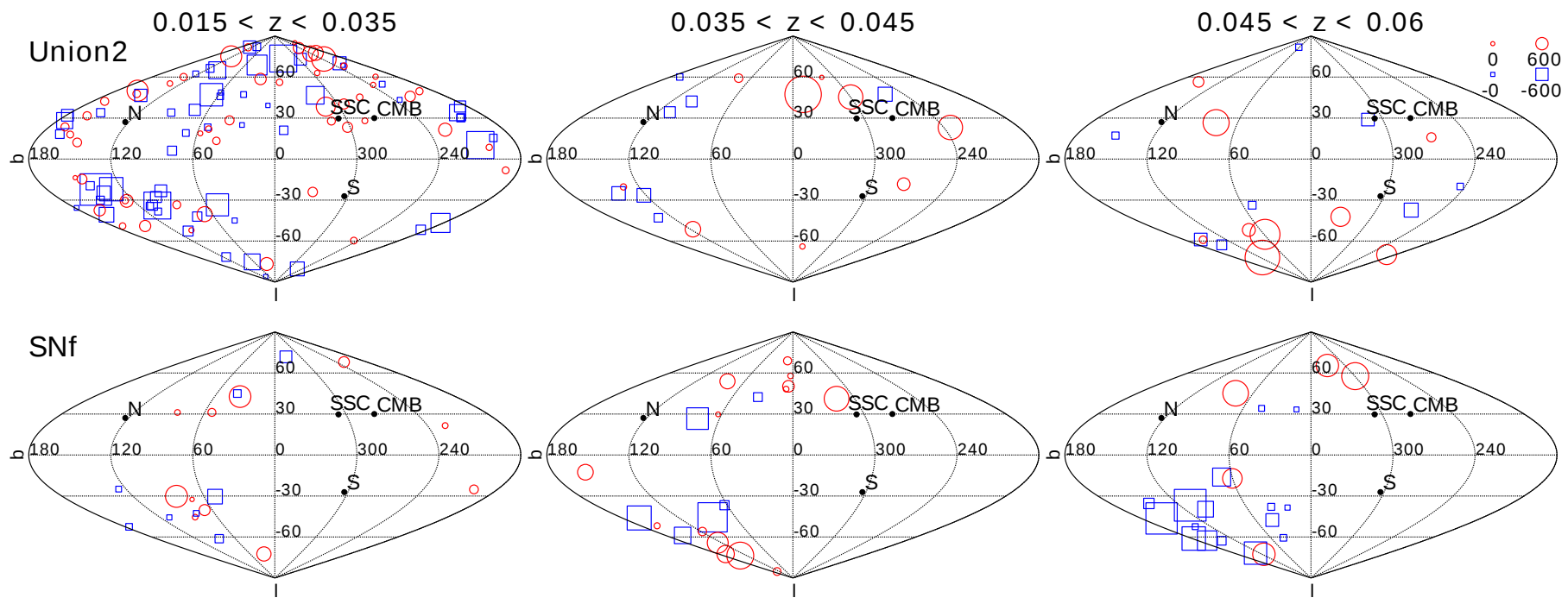

Fig. 1. Peculiar velocities of individual SNe determined from their distance moduli, $\mu_{i}$, by solving Eq. (2) for $v_{\mathrm{DF}}$. The plots show the Union2 (top row) and SNFACTORY (bottom row) datasets in the redshift bins $0.015<z<0.035$ (left column), $0.035<z<0.045$ (middle column) and $0.045<z<0.06$ (right column). The marker diameter of each SN is proportional to the absolute value of the velocity plus an offset (see the scale at the top right), with red circles corresponding to positive velocities and blue squares corresponding to negative ones. For reference, the directions of the CMB dipole and the Shapley supercluster (SSC) are shown.

according to Hui \& Greene (2006). The correlation for a bulk flow is smaller than the full correlation up to $\mathrm{SN}$ separations of $\sim 70 \mathrm{Mpc}$. As these few pairs of SNe are mostly at low redshift, we expect no significant effect on the higher-redshift bins to which we add the most new data.

Here we have restricted the analysis to a dipole in the peculiar velocities. However, a bulk flow due to a massive attractor like the SSC is expected to a have a shear component as well which would be observed in higher multipole orders. An analysis accounting for the quadrupole and octupole as in Macaulay et al. (2011) will be presented in a subsequent paper (Feindt et al., in prep.).

\subsection{Smoothed residuals $(S R)$}

As an alternative means of identifying potential bulk flow motions, the direction on the sky with the highest deviation of the data from the isotropic $\Lambda$ CDM cosmological model is determined. The corresponding test statistic is constructed from the error-weighted Hubble residuals, $r_{i}$, from Eq. (5), i.e.,

$r_{i}=\frac{\mu_{i}-5 \log _{10}\left(d_{\mathrm{L}}^{(0)}\left(z_{i}\right) / 10 \mathrm{pc}\right)}{\sigma_{\mu}}$.

The method of smoothed residuals (SR) as described in detail in Colin et al. (2011) was implemented and further extended for this work. It can be briefly summarized as follows:

The statistic used for such an analysis is

$Q\left(l_{\mathrm{SR}}, b_{\mathrm{SR}}\right)=\frac{\sum_{i} r_{i} \cdot W\left(l_{\mathrm{SR}}, b_{\mathrm{SR}}, l_{i}, b_{i}\right)}{\sum_{i} W\left(l_{\mathrm{SR}}, b_{\mathrm{SR}}, l_{i}, b_{i}\right)}$,

where the window function $W\left(l_{\mathrm{SR}}, b_{\mathrm{SR}}, l_{i}, b_{i}\right) \equiv W_{i}$ reads

$W\left(l_{\mathrm{SR}}, b_{\mathrm{SR}}, l_{i}, b_{i}\right)=\left(2 \pi \delta^{2}\right)^{-1 / 2} \exp \left(-\frac{L\left(l_{\mathrm{SR}}, b_{\mathrm{SR}}, l_{i}, b_{i}\right)^{2}}{2 \delta^{2}}\right)$.

Here $\delta$ is a smoothing parameter, which in the following is chosen as $\pi / 2$ in order to identify dipoles in the Hubble expansion. $\left(l_{i}, b_{i}\right)$ are the coordinates of a SN and $\left(l_{\mathrm{SR}}, b_{\mathrm{SR}}\right)$ those of the direction in which $Q$ is evaluated. $L$ represents the angular distance between two points.

Colin et al. used $Q\left(l_{\mathrm{SR}}, b_{\mathrm{SR}}\right)=\sum_{i} r_{i} \cdot W\left(l_{\mathrm{SR}}, b_{\mathrm{SR}}, l_{i}, b_{i}\right)$ whereas in Eq. (7) a weighting is introduced that is new to this paper. The weighting factor $\left[\sum_{i} W\left(l_{\mathrm{SR}}, b_{\mathrm{SR}}, l_{i}, b_{i}\right)\right]^{-1}$ avoids oversampling of inhomogeneities due to uneven sky coverage, which can lead to artificial bulk flow signals.

The sky populated with any SN Ia dataset is scanned in steps of $10^{\circ}$ in Galactic coordinates $\left(l_{\mathrm{SR}}, b_{\mathrm{SR}}\right)$. For every direction the value of $Q\left(l_{\mathrm{SR}}, b_{\mathrm{SR}}\right)$ is computed according to Eq. (7) and the maximum and minimum values, i.e., $Q_{\max }$ and $Q_{\min }$, and corresponding directions on the sky are recorded. To quantify the deviation from the model prediction the test statistic

$\Delta Q=Q_{\max }-Q_{\min }$

is evaluated. The significance of large $\Delta Q$ is determined by randomly resampling the directions 5000 times; the $p$-value is the fraction of realizations that have a $\Delta Q$ larger than the measured value. The direction is inferred from

$\min \left(p_{\max }, p_{\min }\right)$

with $p_{\max }$ and $p_{\min }$ being the $p$-values with respect to the $Q_{\max }$ and $Q_{\min }$ distributions, respectively. For example, in the $Q_{\min }$ case the $p$-value is of course the fraction of MC measurements that are smaller than the measured $Q_{\min }$.

This algorithm was applied to subsamples of the datasets as described in Sect. 2 in order to study the run of potential anisotropies with redshift.

\section{Results}

Table 1 shows the velocity and direction of the minimum variance bulk motion, as reconstructed with the dipole fit (DF), as well as the amplitude and direction as obtained from the smoothed residuals (SR) method for each redshift shell for Union2, SNFACTORY and the combined dataset. Also shown are 

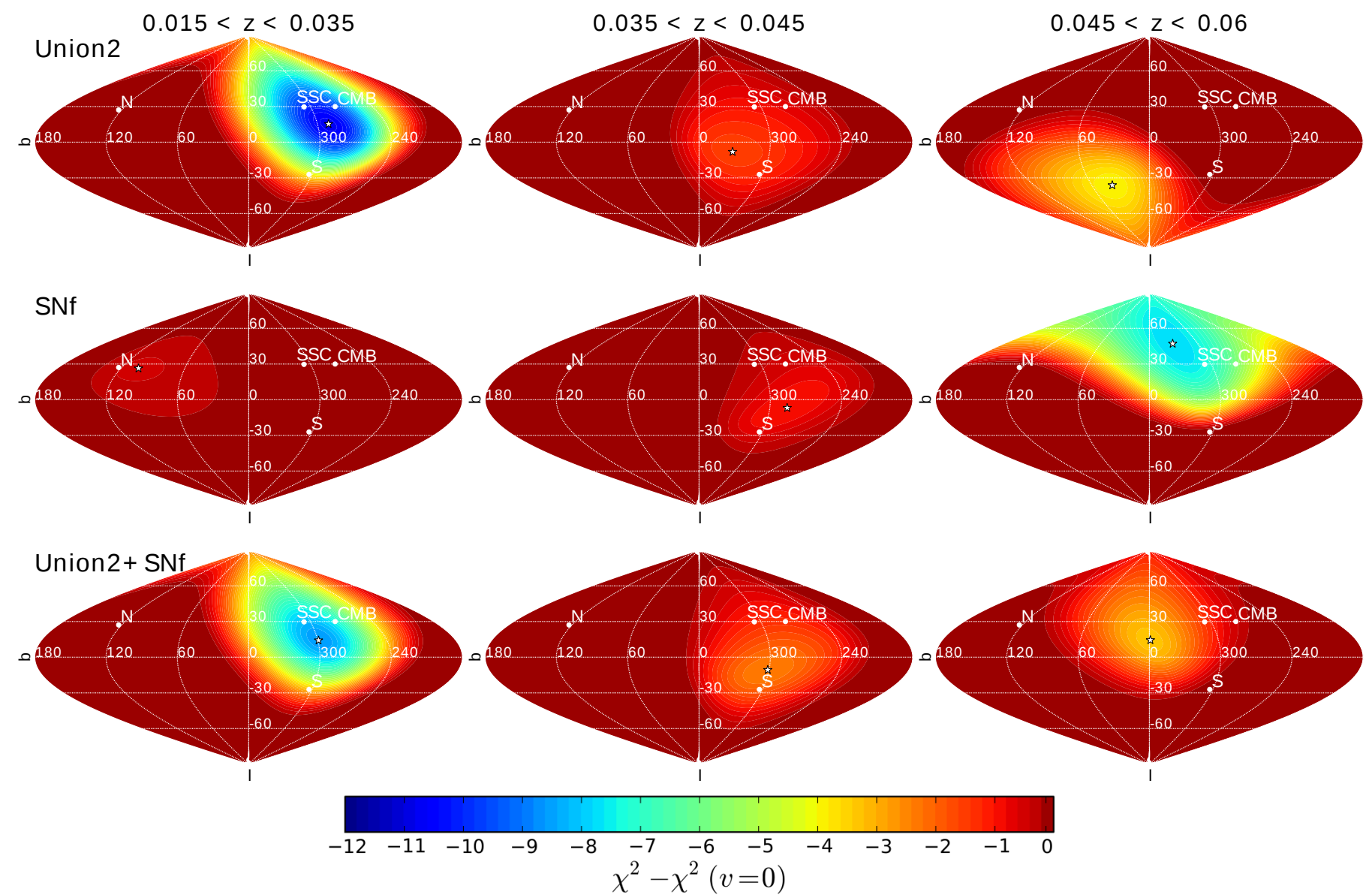

Fig. 2. Variation of $\chi^{2}$ for a dipole fit of SNe Ia from the Union2 (top row) and SNFActoRY (middle row) datasets and their combination (bottom row) as a function of Galactic coordinates $(l, b)$ in the redshift range $0.015<z<0.035$ (left column), $0.035<z<0.045$ (middle column) and $0.045<z<0.06$ (right column). The best fit direction is marked by a star at minimum $\chi^{2}$.

the corresponding $p$-values, i.e., the chance probability to observe a signal of similar or larger significance in a homogeneous and isotropic Universe. In the following, the results of the two analysis methods are presented in more detail.

\subsection{Dipole fit}

We first focus on the nearest redshift shell, $0.015<z<0.035$ $\left(45 h^{-1} \mathrm{Mpc}-105 h^{-1} \mathrm{Mpc}\right)$. The SNfactory targeted higher redshifts than this, thus the constraints here are dominated by data from the Union2 SN compilation. As presented in Table 1, and shown in Fig. 2 there is a flow observed $(p=0.004)$ with a velocity of $v_{\mathrm{DF}}=292 \pm 96 \mathrm{~km} \mathrm{~s}^{-1}$ towards $l=290^{\circ} \pm 22^{\circ}$, $b=15^{\circ} \pm 18^{\circ}$. The combined data show a slightly higher $p$-value of 0.010 than the Union2 dataset alone for $0.015<z<0.035$. While the direction of the bulk flow barely changes, the velocity is slightly lower at $v_{\mathrm{DF}}=243 \pm 88 \mathrm{~km} \mathrm{~s}^{-1}$ for the combined dataset.

However, as there is some tension between the two samples, we need to investigate the consistency of the measured bulk flows to assess whether the samples should be combined in the first place. For this we adopt a scheme similar to the consistency test described by Watkins et al. (2009) by calculating the following $\chi^{2}$ statistics

$\Delta^{2}=\sum_{i, j}\left(\Delta v_{\mathrm{DF}}^{(i)}\right)\left(C^{-1}\right)_{i j}\left(\Delta v_{\mathrm{DF}}^{(j)}\right)$ where $\Delta v_{\mathrm{DF}}^{(i)}$ are the Cartesian components of the vectorial difference between the bulk flow estimates from each sample and $C$ is their combined covariance. For this redshift shell we find $\Delta^{2}=$ 2.62 which corresponds to $p$-value of 0.455 for a $\chi^{2}$ distribution with 3 degrees of freedom. This verifies the observed tension is not significant.

The direction from the combined sample is compatible both with the SSC $\left(l=306.44^{\circ}, b=29.71^{\circ}\right)$ and the CMB dipole $\left(l=276^{\circ} \pm 3^{\circ}, b=30^{\circ} \pm 3^{\circ}\right.$; Kogut et al. 1993). Previous studies have already shown good agreement with the $\mathrm{CMB}$ dipole: e.g., Colin et al. (2011) found a bulk flow of $v_{\text {bulk }}=250_{-160}^{+190} \mathrm{~km} \mathrm{~s}^{-1}$ towards $l=287^{\circ}, b=21^{\circ}$ at a radius of $100 h^{-1} \mathrm{Mpc}$ using a maximum likelihood approach. Watkins et al. (2009) computed $v_{\text {bulk }}=416 \pm 78 \mathrm{~km} \mathrm{~s}^{-1}$ in the direction of $l=282^{\circ}, b=60^{\circ}$ on the same scale. Another general confirmation of these results comes from the study in Lavaux et al. (2010) who report $v_{\text {bulk }}=$ $473 \pm 128 \mathrm{~km} \mathrm{~s}^{-1}, l=220^{\circ}, b=25^{\circ}$ for the $100 \mathrm{~h}^{-1} \mathrm{Mpc}$ frame. Furthermore, Macaulay et al. (2012) find a dipole velocity of $v=$ $380_{-132}^{+99} \mathrm{~km} \mathrm{~s}^{-1}, l=295^{\circ} \pm 18^{\circ}, b=14^{\circ} \pm 18^{\circ}$ and Nusser \& Davis (2011) find a bulk flow of $257 \pm 44 \mathrm{~km} \mathrm{~s}^{-1}$ toward $l=276^{\circ} \pm$ $6^{\circ}, b=10^{\circ} \pm 6^{\circ}$. On the other hand, Courteau et al. (2000) have reported that this shell is at rest with respect to the $\mathrm{CMB}$, finding the bulk flow to be only $70_{-70}^{+100} \mathrm{~km} \mathrm{~s}^{-1}$. Our result is consistent at $1.2 \sigma$ with this amplitude. Therefore the results for nearby flow velocities obtained here are compatible with the literature. Of course the $\mathrm{SNe}$ in this shell are common to several of the studies. 

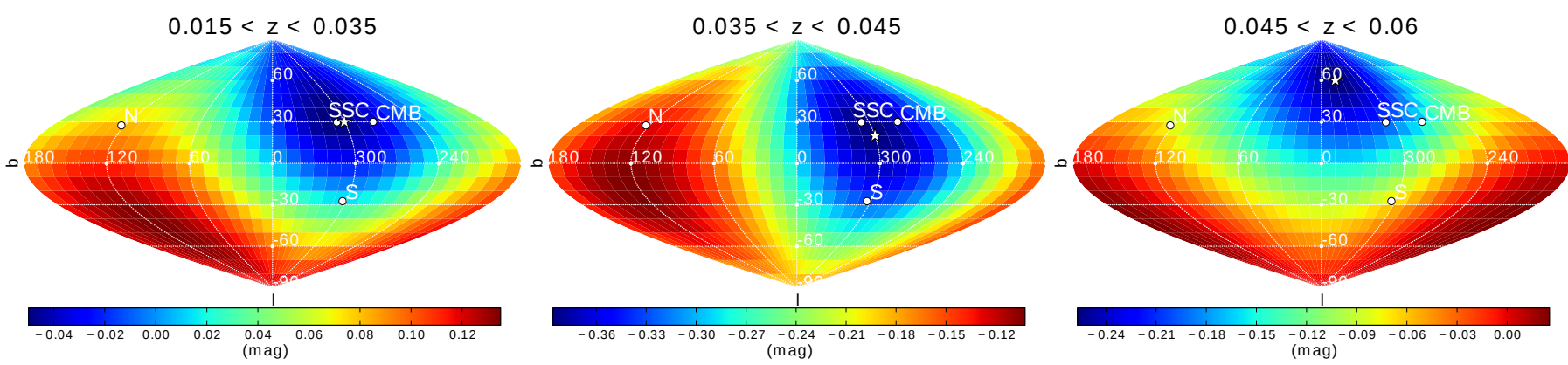

Fig. 3. Magnitude residuals of SNe Ia from the combined Union2 and SNFactory dataset as a function of Galactic coordinates $(l, b)$ after smoothing with a Gaussian window function of width $\delta=\frac{\pi}{2}$ in the redshift range $0.015<z<0.035$ (left), $0.035<z<0.045$ (middle) and $0.045<z<0.06$ (right). The bulk flow direction is marked by a star.

We now turn to the next further redshift shell, $0.035<z<$ 0.045 , where the data from SNFActory starts to dominate the available SN statistics. While the individual datasets show no significant evidence for a bulk motion, the combined datasets lead to a $p$-value of 0.131 for the observed anisotropy to be a random coincidence. While not very significant, it is notable that the best-fit direction is again well aligned with the SSC direction, strengthening the evidence that the observed bulk motion of the shell is real. The upper boundary of the redshift shell was chosen by Colin et al. (2011) such that it intersects the center of the $\mathrm{SSC}^{4}$. Therefore, if the SSC is responsible for the motion, one would expect the flow to retain its direction.

The redshift bin $(0.045<z<0.06)$ intersects the SSC at the lower redshift boundary. If the missing component of the LG velocity is due to the infall into the SSC, one would expect the bulk motion to reverse its direction in the hemisphere around the SSC while it remains the same in the other hemisphere. For the Union2 data, $(0.045<z<0.06)$ the direction of anisotropy appears to be reversed to $l=39^{\circ} \pm 45^{\circ}, b=-36^{\circ} \pm 28^{\circ}$. This is in agreement with the turnover seen by Colin et al. (2011) using the same data. However, with a $p$-value of $p=0.068$ the reversal is not very significant.

The SNFActory data do not support this reversal. Rather, the bulk flow in the corresponding shell maintains its global direction with $l=330^{\circ} \pm 48^{\circ}, b=48^{\circ} \pm 46^{\circ}$ with a rather small $p$-value of 0.006 . The best fit bulk flow velocity $v_{\mathrm{DF}}=$ $941 \pm 390 \mathrm{~km} \mathrm{~s}^{-1}$ appears to be large, but due to its large statistical errors not inconsistent with the bulk flow motions obtained at lower redshifts. When combining the SNFactory and Union2 datasets, this bulk flow beyond the SSC is not as significant as when using SNFACTORY data alone, but still no turnover can be observed: in the shell at $0.045<z<0.06$ the bulk flow is shifted slightly away from this, pointing toward $l=359^{\circ} \pm 32^{\circ}$, $b=14^{\circ} \pm 27^{\circ}$ with a lower velocity of $v_{\mathrm{DF}}=650 \pm 398 \mathrm{~km} \mathrm{~s}^{-1}$.

As with the redshift shell at $0.015<z<0.035$ we observe tension between the datasets in this shell. According to Eq. (11) we find significant tension with $\Delta^{2}=8.59, p=0.035$. We would like to understand the source of this disagreement between the Union2 and SNFACTORY data in the $0.045<z<0.06$ shell. The underlying distribution of SN peculiar velocities on the sky from the Hubble fit is depicted in Fig. 1. The size of the markers correlates with the amplitude of the velocity while color and shape correspond to the direction. When looking at the Union2 data for redshifts $0.045<z<0.06$ (upper right plot) it becomes clear that the putative turnover in flow direction is not exclusively induced by SNe falling into the SSC, as previously suggested by

\footnotetext{
4 The SSC roughly extends from $0.035<z<0.055$ with its center located at $z \sim 0.046$.
}

Colin et al. (2011), but also driven by two distant $\mathrm{SNe}$ in the direction opposite the SSC, SN 1995ac (Riess et al. 1999) and SN 2003ic ${ }^{5}$ (Hicken et al. 2009). In this shell SNe in the vicinity of the SSC should otherwise be significantly blueshifted giving positive residuals, however Union2 lacks SNe there. As a result, we find that the Union2 compilation has insufficient area coverage in this critical redshift shell to address the question of whether the SSC is the source of the CMB-dipole. For the SNFACTORY sample, on the other hand, there are two redshifted SNe that show large residuals $(\sim 1.5 \sigma)$ behind the SSC while $\mathrm{SNe}$ in the opposite part of the sky are mostly blueshifted.

We tested the effect of removing either or both of the two Union2 SNe in question (1995ac and 2003ic). When removing one of them at a time, we find that the tension decreases to $\Delta^{2}$-values of $6.37(p=0.095)$ and $6.32(p=0.097)$ respectively. Removing both decreases the tension to $\Delta^{2}=$ $4.15, p=0.246$. Without SNe 1995ac and 2003ic the amplitude of $v_{\mathrm{DF}}$ determined from Union2 (cf. Table 1) alone decreases to $498 \pm 573 \mathrm{~km} \mathrm{~s}^{-1}$ while its direction shifts to $l=35^{\circ} \pm 66^{\circ}, b=$ $-20^{\circ} \pm 28^{\circ}$. The effect of removing both $\mathrm{SNe}$ on the combined dataset is insignificant as we find $v_{\mathrm{DF}}=629 \pm 336 \mathrm{~km} \mathrm{~s}^{-1}$ towards $l=351^{\circ} \pm 40^{\circ}, b=37^{\circ} \pm 37^{\circ}$. Furthermore we note that the probability of finding a $p$-value of less than 0.035 for at least one of four measurements is $13.3 \%$ and hence finding this tension in one of four redshift shells is not as significant as it may seem by itself.

Finally, for the $0.06<z<0.1$ shell, no bulk motion is detected for any of the samples - the best fit velocity is consistent with zero with an associated uncertainty of $\sim 400 \mathrm{~km} \mathrm{~s}^{-1}$. This contradicts the results of Kashlinsky et al. (2010) who measured a bulk flow of $\sim 1000 \mathrm{~km} \mathrm{~s}^{-1}$ aligned with the CMB dipole direction at the same distance. For a better comparison to their results, we fit the absolute value of the dipole velocity while keeping its direction fixed at the CMB direction. This yields a dipole velocity of $26 \pm 236 \mathrm{~km} \mathrm{~s}^{-1}$, i.e. we rule out a bulk flow as seen by Kashlinsky et al. at $\sim 4 \sigma$.

\subsection{Smoothed residuals analysis}

The results for the smoothed residual analysis can be found in the second column to the right of Table 1 . The magnitude residuals for the combined dataset for $0.015<z<0.06$ are shown in Fig. 3. The directions determined by this method are fully compatible in reconstructed bulk flow directions and significance

5 This SN has been previously identified as an outlier in the hostluminosity relation (Kelly et al. 2010). Furthermore we determined that it is located in the galaxy cluster Abell 0085. The difference in redshifts is insignificant $(\Delta z=0.00007)$. 
levels with what was already found in the dipole fit. Apparent differences occur in bins with poor statistics where basically noise is fit and the error bars are correspondingly large.

Comparing the directions determined for the Union2 sample to those of Colin et al. (2011), we find agreement within $\sim 15^{\circ}$ for most shells for which we find $p<0.5$. The scientifically most interesting shell is that at $0.045<z<0.06$. We find an insignificant deviation of $15^{\circ}$ in that shell. However, note that there are two differences between our analysis and that of Colin et al. (2011). Firstly, we divide their test statistic $Q$ by the sum of the weights $W_{i}$ to avoid artificially large values in regions of the sky where more SNe were observed. Secondly, we set the redshifts of SNe near galaxy clusters to the cluster redshifts. One of these SNe is SN 19930 in the cluster Abell $3560(z=0.04985)$. As its redshift provided in the Union2 compilation is $0.0529^{6}$, the $\mathrm{SN}$ appears to be moving toward us instead of away from us when using the redshift of Abell 3560. This increases the statistic $Q$ for directions opposite to that of the SSC. However, if we use the redshifts that are provided in the Union2 compilation, we find a minimum of $Q$ at $l=50^{\circ}, b=-10^{\circ}$, which deviates from the result of Colin et al. by $37^{\circ}$. Therefore our implementation of the smoothed residuals is indeed not biased by regions of larger sample density.

Furthermore a larger disagreement between the methods is found at redshift $0.1<z<0.4$ where SNe from SDSS (Holtzman et al. 2008), which are located on a thin stripe on the sky, dominate the dataset.

\subsection{Systematic uncertainties}

Systematic errors associated with $\mathrm{SNe}$ Ia as distance indicators have been scrutinized carefully for the measurement of the expansion history of the Universe. Some of the main sources of redshift- or sample-dependent systematic error are connected to flux zero points, $K$-corrections and Malmquist bias (Amanullah et al. 2010; Regnault et al. 2009). They can also conspire in non-trivial ways, such as in the case of the "Hubble bubble" (Jha et al. 2007), an apparent 6\% increase of the local Hubble parameter. The Hubble bubble was explained in Conley et al. (2007) as the result of a combination of a bias in color, i.e., the closest $\mathrm{SNe}$ in the sample show more reddening, and the choice of the reddening correction parameter $\beta$. Below we discuss our measurement of bulk flows in the context of (1) a redshift or sample-dependent systematic error on the distance modulus and (2) direction-dependent systematic errors.

To first order, a redshift-dependent systematic error averages out when inferring directional bulk flows. It is only because of e.g., anisotropic sky coverage, that the averaging process can result in a residual bias. For the bulk flow analyses the distribution of $\mathrm{SNe}$ on the sky has a great impact on the sensitivity in a given direction, e.g., if most observations lie in a direction perpendicular to the bulk flow, no good constraints on its value can be derived. Alternatively if a SN sample that lies in a preferred direction has a systematic bias, this will induce a false bulk flow signal.

To quantify this effect, we calculate the weighted mean direction $\overline{\boldsymbol{n}}$ of the datasets, i.e.,

$\overline{\boldsymbol{n}}=\frac{\sum_{i} w_{i} \boldsymbol{n}_{i}}{\sum_{i} w_{i}} \quad$ with $\quad w_{i}=\frac{1}{\sigma\left(\mu_{i}\right)^{2}} \frac{d_{\mathrm{L}}^{(\mathrm{dipole})}\left(z_{i}, v_{\mathrm{DF}}\right)}{d_{\mathrm{L}}^{(0)}\left(z_{i}\right)}$

\footnotetext{
6 This redshift differs from the one obtained from NED $(z=0.514$ in CMB restframe).
}

where $\boldsymbol{n}_{i}$ is the normal vector for a SNe's coordinates. The weights, $w_{i}$, are calculated from the uncertainty $\sigma\left(\mu_{i}\right)$ of the distance modulus and the ratio of $d_{\mathrm{L}}^{\text {(dipole) }}$ to $d_{\mathrm{L}}^{(0)}$ (see Eq. (2)) for an arbitrary velocity where the latter corresponds to the fact that in our weighting scheme SNe at higher redshifts have a smaller weight on the inferred bulk motion. Note that the dependence of $d^{\text {(dipole) }}$ on $v_{\mathrm{DF}}$ cancels when dividing by the sum of the weights, as it is linear in $v_{\mathrm{DF}}$. For a perfectly homogeneous distribution of observations $\overline{\boldsymbol{n}}$ would vanish. In that case the sources of systematic errors in cosmological analyses would average out in the measurement of the bulk flow. If, on the other hand, the amplitude of $\overline{\boldsymbol{n}}$ is non-zero, the systematic effects would shift proportionately the resulting bulk flow in the direction of $\overline{\boldsymbol{n}}$.

It can be expected that the different SN samples, which where obtained with different telescopes and analysis pipelines, will have a different degree of bias, i.e., all brighter or dimmer than expected. Amanullah et al. (2010) has investigated such biases within the Union2 compilation, and at the level of the statistical errors that range from 0.01 to 0.05 magnitudes, has not found evidence for their presence. Furthermore the effect of redshift-dependent systematic uncertainties, e.g., reference star colors, which are the largest contributor to the systematic uncertainties (see Amanullah et al. 2010), is considerably smaller because the portion of our SN sample with a statistical weight spans a small redshift range. Assuming that all $\mathrm{SNe}$ in a redshift shell are biased by $\delta m$ magnitudes, the resulting bias in velocity would be $\delta \boldsymbol{v}\left[\mathrm{km} \mathrm{s}^{-1}\right] \approx 1.3 \times 10^{5}$. $z \cdot \delta m \cdot \overline{\boldsymbol{n}}$. Thus, for the lowest redshift bin $(|\overline{\boldsymbol{n}}|=0.252)$, assuming a bias of 0.03 magnitudes, one would obtain a velocity bias of $\sim 25 \mathrm{~km} \mathrm{~s}^{-1}$. For the redshift shell $0.035-0.45$, one would obtain a velocity bias of $\sim 33 \mathrm{~km} \mathrm{~s}^{-1}$, while for the redshift bins $0.045-0.06$ and $0.06-0.1$ one would obtain a velocity bias 70 and $100 \mathrm{~km} \mathrm{~s}^{-1}$, respectively. This potential bias should be put into context of the statistical error ${ }^{7}$. For all shells it can be seen that such a bias would be smaller than the statistical error. Note also that the bias is a vector quantity and therefore will have the strongest effect on the velocity if it is parallel or antiparallel to the bulk velocity - otherwise it will mainly change the direction.

To absorb any bias that may be present due to a constant shell-dependent magnitude offset, we added a monopole term to the luminosity distance in Eq. (2):

$d_{\mathrm{L}}\left(z, v_{\mathrm{m}}, v_{\mathrm{DF}}, \theta\right)=d_{\mathrm{L}}^{(0)}(z)+d_{\mathrm{L}}^{(\text {monopole })}\left(z, v_{\mathrm{m}}\right)+d_{\mathrm{L}}^{(\mathrm{dipole})}\left(z, v_{\mathrm{DF}}, \theta\right)$

This term corresponds to a constant radial velocity $v_{\mathrm{m}}$ in the redshift shell that is analyzed and $d_{\mathrm{L}}^{\text {(monopole) }}$ is calculated exactly as $d_{\mathrm{L}}^{\text {(dipole) }}$ according to Eq. (4) but without the dependence on $\cos (\theta)$. For the SN magnitudes this translates to an equal shift of all luminosities in that bin, while a dipole term causes $\mathrm{SNe}$ in one half of the sky to appear brighter than expected from their redshift and those on the other half to appear fainter. We generally find monopole velocities that are consistent with zero and all effects on the dipole velocities are within $1 \sigma$ of the fit without a monopole. In particular for the 1 st and 4th bin, the best fit velocities have essentially not changed, indicating the absence of a potential bias due to a magnitude offset. For the case of the 4th bin, where we fixed the direction to the CMB dipole in order to compare to Kashlinsky et al. (2010), the dipole velocity

7 The achievable statistical error can be approximated as $\sigma_{v}\left[\mathrm{~km} \mathrm{~s}^{-1}\right] \approx$ $3.6 \times 10^{4} \cdot z \cdot N_{\mathrm{SN}}^{-1 / 2}$, where $N_{\mathrm{SN}}$ is the number of SNe. We have assumed an isotropic distribution and a distance modulus uncertainty of 0.15 . 
changes from $26 \pm 236 \mathrm{~km} \mathrm{~s}^{-1}$ for a fit without monopole term to $-32 \pm 271 \mathrm{~km} \mathrm{~s}^{-1}$ with monopole term.

As SN statistics for redshifts $0.03<z<0.1$ are still low, one needs to be careful when combining two datasets with $\bar{n}$ pointing in opposite directions. For the combination of the Union2 and SNFACTORY datasets, we have therefore checked that this is not the case, finding that the angles between $\overline{\boldsymbol{n}}$ do not exceed $\sim 90^{\circ}$ for all shells (see the rightmost column of Table 1). Additionally the amplitudes of $\overline{\boldsymbol{n}}$ are smaller than 0.5 for all shells with $z<0.1$ and smaller than 0.35 for the combined data. For higher redshifts the absolute value can be larger, especially for $0.1<z<0.4$ because the SNe from SDSS (Holtzman et al. 2008) are clustered in one direction.

We also tested alternative corrections of the SN magnitude (Eq. (1)) that we can apply to the SNfactory dataset. As was shown in Bailey et al. (2009), using the ratio of spectral flux at $642 \mathrm{~nm}$ and $443 \mathrm{~nm}$ reduces the intrinsic scatter further than using the SALT2 light-curve fit parameters $x_{1}$ and $c$. We applied this correction to 93 of the 117 SNFACTORY SNe and compared the results with those for the SALT2-corrected distance moduli of the same $\mathrm{SNe}$, finding the changes to be below $1 \sigma$ for the direction and below $0.2 \sigma$ for the bulk flow velocity.

Recent studies have shown that the standardized magnitudes of SNe Ia are correlated with the properties of their host galaxies, such that $\mathrm{SNe}$ in galaxies with higher stellar mass $M_{*}$ are brighter on average (Kelly et al. 2010; Sullivan et al. 2010; Lampeitl et al. 2010; Childress et al. 2013b). To test the impact of this on our analysis, we implemented a mass step function, i.e., we split the SNFactory dataset at $\log \left(M_{*} / M_{\odot}\right)=10$ (using host mass data presented in Childress et al. 2013a) and allowed for different normalizations of the split samples. There is no change in our results for the bulk flow (less than $0.2 \sigma$ for the bulk flow velocity.) Similarly, we tested extending the SALT2 correction by adding the logarithm of the host metallicty from Childress et al. (2013a). This limited the dataset to $68 \mathrm{SNe}$. Again our results did not change significantly (by less than $0.1 \sigma$ for both bulk flow direction and velocity).

Finally, we turn to explicitly direction-dependent systematic errors. As the effect of extinction by dust in the Galaxy is anisotropic, it is expected to have a larger contribution to the systematic error of the bulk flow than on cosmological parameters. To assess the effect of such uncertainties, we increased the distance modulus of each SN by $10 \%$ of the reported extinction in its direction (Schlegel et al. 1998). This changes the inferred bulk flow and its direction by less than $0.05 \sigma$. Therefore Milky Way extinction can be considered a small source of systematic uncertainties for these data sets. Improper atmospheric extinction corrections can also lead to a directional bias. However, it is usually an integral part of a calibration procedure that relies on few assumptions, resulting in a small contribution to the total magnitude error (see, e.g., Regnault et al. 2009; Buton et al. 2013) that can be safely neglected for this work.

\section{Searching for matter overdensities}

Applying the dipole fit (DF) method as well as the method of smoothing residuals (SR) to the datasets has shown a bulk flow that continues to point towards the $\mathrm{CMB}$ dipole direction and extends beyond the SSC. A reversal of the bulk flow direction at the distance of the SSC was rejected (see Table 1 and Fig. 2 lower panel) in the SNFACTORY and combined data, while the apparent backside infall in Union2 was identified to stem from two $\mathrm{SNe}$ located in the opposite direction of the SSC that are redshifted, instead of blueshifted. In what follows, we chose an alternative, model-dependent approach to quantify the constraints of the SN data on an SSC-like attractor scenario. We thereby test for the presence of a single, massive object along the line of sight of the SSC. We restrict our study to the simplified model of a spherical attractor of constant density $\rho$ with a radius of $R=50 \mathrm{Mpc}$, which therefore has a total mass of

$M_{\text {attractor }}=\frac{4 \pi R^{3}}{3} \rho$.

Because the observed bulk motion at larger redshifts is only marginally significant, this approach is chosen in favor of a more conventional reconstruction of the entire galaxy density field (as in, e.g., Erdoğdu et al. 2006; Lavaux et al. 2010) which would require larger $\mathrm{SN}$ statistics for meaningful results.

Furthermore, we are interested in measuring the overdensity at the distance of the SSC and beyond where we contribute the most new data. At lower redshifts, where the overdensities have already been mapped, our model will be much less accurate as we do not include known (but less massive) structures.

In general, local variations in density, $\rho(\boldsymbol{r})$ (where $\boldsymbol{r}$ is a position vector of proper distance originating at the attractor), will result in additional contribution to the redshift. Perturbing the distance modulus $\mu=5 \log \left(d_{\mathrm{L}}^{(0)}\right.$ ) (with $d_{\mathrm{L}}^{(0)}$ as per Eq. (3)) by an extra peculiar velocity induced redshift $z_{\mathrm{p}}$ yields a measured distance modulus ${ }^{8}$

$\mu_{i}=\mu\left(z_{i}-z_{\mathrm{p}}\right)$.

The peculiar velocity $v_{\mathrm{p}}$ of a single $\mathrm{SN}$ in the gravitational field of an overdensity $\delta(\boldsymbol{r})=\left(\rho(\boldsymbol{r})-\rho_{\mathrm{c}} \Omega_{\mathrm{M}}\right) / \rho_{\mathrm{c}} \Omega_{\mathrm{M}}$ according to Peebles (1993) and Muñoz \& Loeb (2008) is given by

$$
\begin{aligned}
\boldsymbol{v}_{\mathrm{p}}(\delta, \boldsymbol{x}) & =\frac{a f H}{4 \pi} \int \frac{\boldsymbol{y}-\boldsymbol{x}}{|\boldsymbol{y}-\boldsymbol{x}|^{3}} \delta(\boldsymbol{y}) \mathrm{d}^{3} \boldsymbol{y} \\
& \approx \frac{\Omega_{\mathrm{M}}^{0.55} H_{0}}{4 \pi(1+z)} \int \frac{\boldsymbol{y}-\boldsymbol{x}}{|\boldsymbol{y}-\boldsymbol{x}|^{3}} \delta(\boldsymbol{y}) \mathrm{d}^{3} \boldsymbol{y}
\end{aligned}
$$

where $\boldsymbol{x}$ and $\boldsymbol{y}$ are the proper distance vectors pointing from the center of gravity of the overdensity to a particular SN and to each mass element of the attractor, respectively, and $\rho_{\mathrm{c}}$ is the critical density of the universe. Projecting $\boldsymbol{v}_{\mathrm{p}}$ along the line of sight the resulting additional redshift is given by

$z_{\mathrm{p}}(\delta, \boldsymbol{x})=-\frac{v_{\mathrm{p}}(\delta)}{c} \frac{\boldsymbol{x}+\boldsymbol{s}}{|\boldsymbol{x}+\boldsymbol{s}|} \cdot \frac{\boldsymbol{s}}{|\boldsymbol{s}|}$

where $s$ denotes the vector between the observer and a SN.

In this study $\delta(\boldsymbol{r})$ is assumed to be constant within a sphere with radius $R=50 \mathrm{Mpc}$ corresponding to the size of the SSC. Therefore the total mass of the attractor is given by rewriting Eq. (14) as

$M_{\text {attractor }}=\frac{4 \pi R^{3}}{3} \rho_{\mathrm{c}} \Omega_{\mathrm{M}}(1+\delta)$.

The overdensity profile within the sphere is of little importance to our analysis as most $\mathrm{SNe}$ are outside its boundary. However, as a single $\mathrm{SN}$ inside the sphere can have a large effect on the results, we introduce weights, $w_{i}$, that are defined as

$w_{i}=\left\{\begin{array}{ll}\sin ^{16}\left(\frac{\pi}{2} \frac{r_{i}}{R}\right) & \text { if } r_{i}<R \\ 1 & \text { if } r_{i} \geq R\end{array}\right.$,

8 For an additional (positive) redshift $z_{\mathrm{p}}$ the measured distance modulus at $z$ is actually smaller than predicted. 


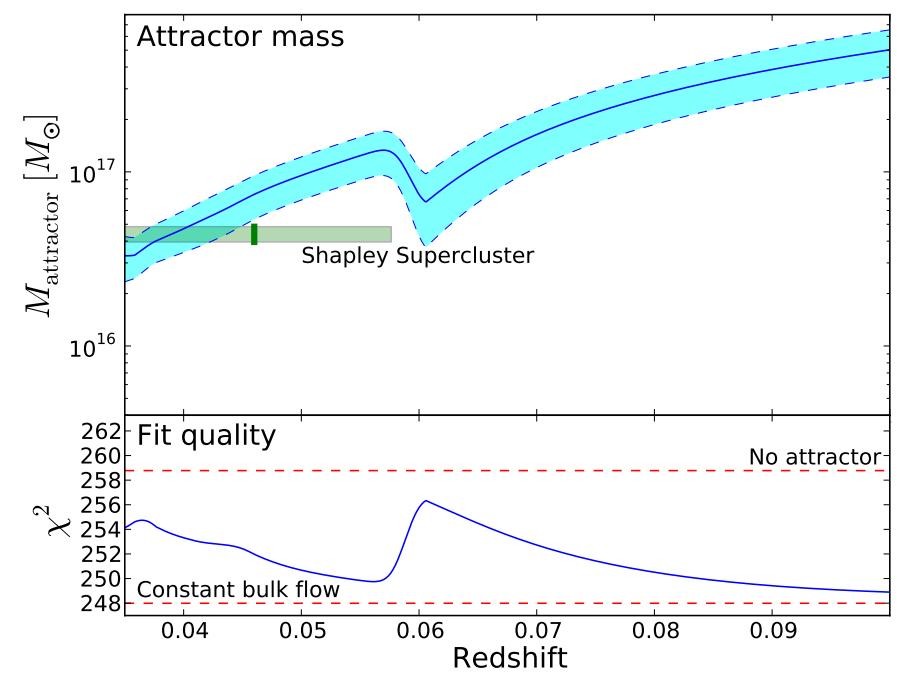

Fig. 4. Attractor mass $M_{\text {attractor }}$ as a function of redshift $z$ accounting for the SNe Ia peculiar velocities in the combination of the Union2 and SNFACTORY datasets along with $\chi^{2}$ fitting via Eq. (20). The blue shaded area shows the uncertainty of the mass determined for a given attractor redshift. The green box shows the mass range as well as the approximate size of the SSC from Muñoz \& Loeb (2008). The upper red dashed line shows the $\chi^{2}$ value for assuming no attractor while the lower one shows the $\chi^{2}$ value for a constant bulk flow.

where $r_{i}$ is the distance from the center of the overdensity. The overdensity, $\delta(\boldsymbol{r})$, is determined by minimizing the expression

$\chi^{2}=\sum_{i} \frac{\left|\mu_{i}-\mu\left(z_{i}-z_{\mathrm{p}}(\delta)\right)\right|^{2}}{\sigma_{\mu}^{2}} w_{i}^{2}$.

Varying the redshift of such a hypothetical concentration along the direction $\left(l_{\mathrm{SSC}}, b_{\mathrm{SSC}}\right)$ towards the SSC and minimizing the expression in Eq. (20) yields the corresponding overdensity at a redshift $z$, i.e., $\delta=\delta\left(z, l_{\mathrm{SSC}}, b_{\mathrm{SSC}}\right)$, necessary to account for the peculiar motions (see Eq. (16)) present in the data.

In Fig. 4 the required attractor mass for the peculiar velocity field extracted from all $\mathrm{SNe}$ at $z<0.1$ in the combination of the Union2 and SNFACTORY datasets is shown as a function of redshift $z$ in the direction of the SSC. As the overdensities at lower redshift have already been mapped, we restricted the redshift of the model attractor to $0.035<z<0.1$. Additionally, the corresponding $\chi^{2}$-values for the fit with Eq. (20) are shown as a measure of the fit quality. A mass placed at large distances asymptotically approaches the case of a constant bulk velocity. This is because an attractor at larger distance leads to a more homogeneous peculiar velocity field (and thus less shear) in the nearby Universe. We find that a mass concentration in the proximity of the SSC is only marginally consistent with our data. While an attractor at the location of the SSC $(z \sim 0.045)$ is disfavored at a level of $2.1 \sigma\left(\Delta \chi^{2}=4.4\right)$, compared to a constant bulk velocity determined by a dipole fit of the same data ${ }^{9}$, this $\Delta \chi^{2}$ decreases rapidly with redshift and disagrees by less than $2 \sigma$ at redshifts $0.046<z<0.058$. This conclusion is based purely on the shape of the gravitational field, and not the mass itself. The mass that is obtained for the location of the SSC is larger by a factor of two or more compared to current mass estimates

\footnotetext{
9 This result does not contradict our rejection of a dark flow in Sect. 4 because this analysis uses all $\mathrm{SNe}$ at $z<0.1$ and therefore $\mathrm{SNe}$ at low redshifts contribute to it.
}

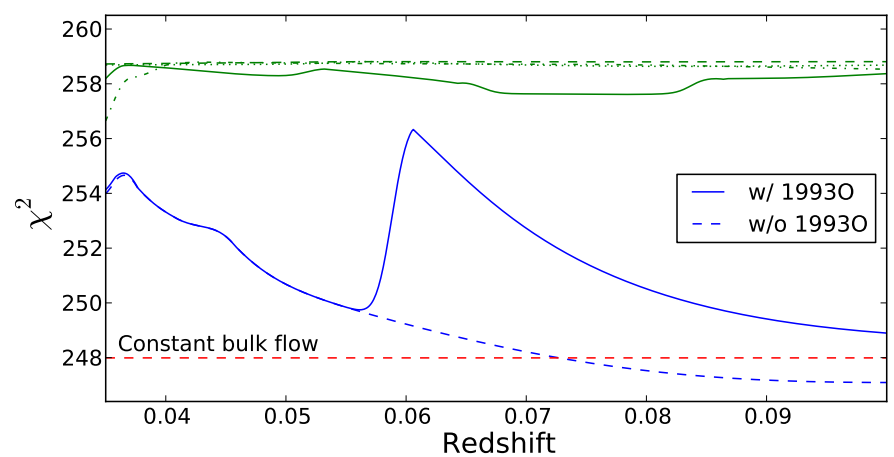

Fig. 5. $\chi^{2}$ values for attractor fits in the direction of the SSC with and without SN 1993 O (blue lines) as well as for directions $90^{\circ}$ north (solid green), south (dotted green), east (dashed green) and west (dash-dotted green) of this in Galactic coordinates.

of the SSC (Muñoz \& Loeb 2008; Sheth \& Diaferio 2011) ${ }^{10}$, suggesting the need for an additional gravitational source.

An attractor mass at a redshift further out leads to better fit results, however. The required overdensity thereby asymptotically grows with the square of the distance, i.e., $\delta \propto d^{2}$. We have investigated if the SSC direction is indeed a preferred direction by repeating the fit placing the attractor in directions that are perpendicular to the SSC direction. Figure 5 shows the evolution of the $\chi^{2}$ values for these directions that show no significant decrease for any attractor distance. Therefore a search focused on an attractor in the SSC direction is fully justified.

Furthermore, we tested whether the assumed radius of the attractor influences the fit results. As the velocity field outside the overdensity only depends on the total mass of the attractor, we expect this influence to be small. However, as the velocity field inside is sensitive to the radius, $\mathrm{SNe}$ inside the overdensity can affect the fit results. Five $\mathrm{SNe}$ are within the boundary of the attractor for some redshifts but their effect is limited by the deweighting according to Eq. (19). Only for SN 19930 does the effect remain noticeable as a dip in the $\chi^{2}$ values and a bump in the attractor mass around $z \sim 0.05$, both of which become narrower for smaller radii. SN 19930 is located in the galaxy cluster Abell $3560(z=0.04985), 28 \mathrm{Mpc}$ from the center of the SSC and thus within the assumed spherical overdensity. For an attractor radius of $50 \mathrm{Mpc}$ only the high-redshift edge of the $\chi^{2}$ dip is visible as a large increase in the $\chi^{2}$ value around at $z \sim 0.06$. This is not only caused by the SN being inside the boundary of the attractor but rather by its proximity to the attractor in general. SN 19930 appears to be moving toward us and therefore an attractor placed behind it will lead to a much larger residual than an attractor in front of it. This effect is not seen immediately at the redshift of the SN because it is suppressed by the weights, $w_{i}$, while the $\mathrm{SN}$ is inside the overdensity. Removing SN 19930 from the dataset also removes the $\chi^{2}$ step as well as the corresponding step in the attractor mass. For the SSC location $(z=0.045)$ variation of the SSC size or the exclusion of SN 19930 has little effect on the fit quality as the residual for SN 19930 is almost zero.

In order to better understand the limitations of our simplified model we calculated the impact of inserting well-known major underdensities. We first mimic the Local Void, then the Boötes Void, finding only $5 \%$ variations in the best fitting attractor mass and changes in $\Delta \chi^{2} \ll 1$. Likewise, older work based on the Abell Cluster Catalog (Scaramella 1995), or more modern works

10 Lavaux \& Hudson (2011) found a higher values for the mass of the SSC assuming a larger radius of the SSC. 


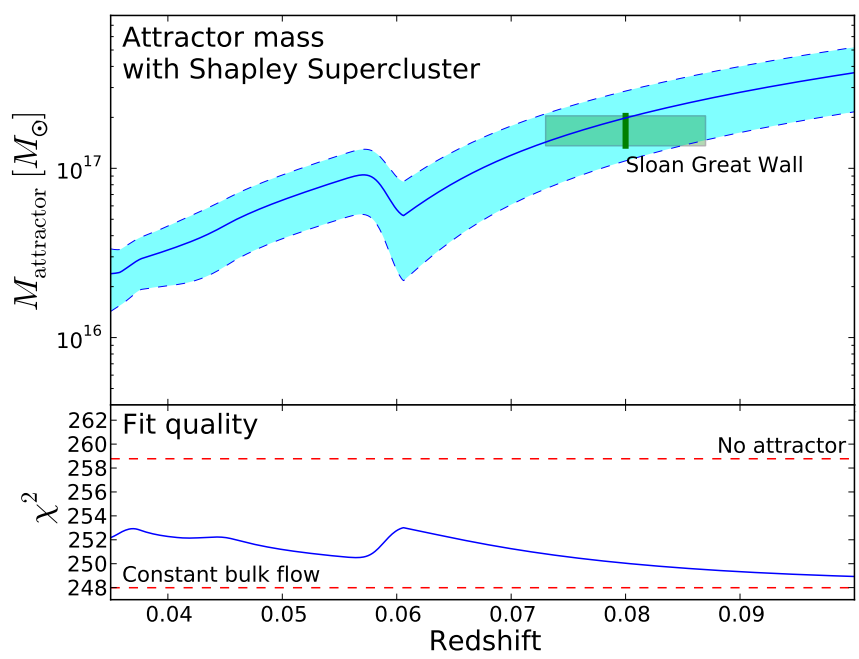

Fig. 6. Attractor mass $M_{\text {attractor }}$ as a function of redshift $z$ as in Fig. 4 where an attractor of SSC mass and size $\left(4.4 \times 10^{16} M_{\odot}, 50 \mathrm{Mpc}\right)$ was inserted at $z=0.045$. The green box shows the mass range as well as the approximate size of the Sloan Great Wall from Sheth \& Diaferio (2011).

using very carefully constructed X-ray selected cluster catalogs (Kocevski et al. 2007) reinforce the view that the SSC is the major attractor within the redshift range of our supernova sample. Thus, while simple, the model we employ is sufficient for the purposes of exploring attractors in the direction of the SSC.

To test for an attractor in addition to the SSC, we have repeated the fit with the SSC inserted as a fixed overdensity of mass $4.4 \times 10^{16} M_{\odot}$ and radius $50 \mathrm{Mpc}$ at $z=0.045$ (see Fig. 6). For this mass fit the local minimum of the $\chi^{2}$ value near the SSC location becomes shallower, while the preference for a distant attractor remains. It was shown in Sheth \& Diaferio (2011) that a single supercluster of the mass of the SSC can still be expected for a sphere of $200 h^{-1} \mathrm{Mpc}$ radius. An additional more distant supercluster seems an unlikely explanation, since it would need to be rather massive and hence would be rare: extrapolating the mass function for superclusters from Lim \& Lee (2013), we find that, e.g., a factor of 3 more massive cluster would already be $\sim 50$ times rarer. A better explanation might be noncollapsed structure. The Sloan Great Wall - the largest known structure in the universe - is located near the direction of the SSC, at a redshift $z=0.07-0.08$. Its mass is estimated to be $1.2 \times 10^{17} h^{-1} M_{\odot}$ by Sheth \& Diaferio (2011) and thus, together with the SSC, could explain the size of the bulk flow. However, since it is an extended source and not perfectly aligned with the SSC, our analysis cannot be directly applied. A full analysis will be performed elsewhere (Feindt et al., in prep.).

\section{Conclusion}

We have investigated two independent samples of SNe Ia - the Union2 data as well as 117 new SNe from the SNFACTORY for anisotropies in the expansion rate. The data were divided into redshift shells and for each shell we obtain the degree of anisotropy by fitting for a bulk velocity of the shell, on top of the expansion expected within a baseline homogeneous isotropic universe with a flat $\Lambda \mathrm{CDM}$ metric. Our key findings are:

- for the lowest redshift bin $(0.015<z<0.035)$, bulk motion was detected at $2.3 \sigma$ in the direction of the CMB dipole, consistent with previous findings. These constraints are dominated by the Union 2 data;
- for the intermediate redshift bin $(0.035<z<0.045)$, bulk motion was observed with $1.1 \sigma$ significance, consistent with the directions of the CMB dipole and the SSC. Only SNFACTORY and Union2 data combined show the observed weak evidence; by themselves the datasets show no significant trends;

- for the next higher redshift bin $(0.045<z<0.06)$, bulk motion was detected at $2.6 \sigma$ significance in the direction of the CMB dipole analyzing SNFActory SNe. This reduces to $1.4 \sigma$ if one combines them with the Union2 SNe. The constraints in this bin are driven by the SNFActory data;

- we have shown that an attractor in the proximity of the SSC is only marginally consistent with our data and that the required attractor mass is larger than previous measurements of the SSC mass, suggesting the need for another gravitational source;

- for the highest redshift bin $(0.06<z<0.1)$ for which we can obtain meaningful constraints we find a bulk velocity that is consistent with zero, with a statistical uncertainty of around $400 \mathrm{~km} \mathrm{~s}^{-1}$. Limiting the direction to that of the CMB dipole, the uncertainties are reduced to around $240 \mathrm{~km} \mathrm{~s}^{-1}$. Therefore we rule out (at $\sim 4 \sigma$ ) a dark flow as reported by Kashlinsky et al. (2010) (see below);

- for all redshift shells analyzed, statistical errors dominate over systematic errors. The significance of the identified anisotropies was reproduced with an alternative analyses based on the smoothed residual method Colin et al. (2011).

The tentative observation, that the dipole motion does not appear to converge on a distances scale of the SSC, i.e., $150 h^{-1} \mathrm{Mpc}$, begs for an explanation. Within $\Lambda \mathrm{CDM}$ structure formation one can expect bulk flows reaching to distances beyond the ones observed here (see, e.g., Nusser \& Davis 2011), a possibility that will be explored in more detail elsewhere (Feindt et al., in prep.).

Other possible explanations for the observed anisotropies are related to the CMB rest frame. Wiltshire et al. (2013) argue in favor of differences in the distances between the observer and the surface of last scattering due to nearby foreground structures mimicking an additional boost. More perplexing would be the presence of a dark flow, i.e., a coherent flow extending throughout the visible Universe, possibly originating in pre-inflation space-time (Kashlinsky et al. 2008). The case for a dark flow is motivated by recent observation of coherent motion of galaxy clusters, deduced from the kinematic Sunyaev-Zeldovich effect (Kashlinsky et al. 2010). Bulk flow velocities of $\sim 1000 \mathrm{~km} \mathrm{~s}^{-1}$ aligned with the CMB dipole were obtained for shells with radius ranging from $270 \mathrm{Mpc}$ to $800 \mathrm{Mpc}$. While it would be another remarkable property of our Universe, their results rely on a new method, and hence require confirmation. Turnbull et al. (2012) already derived an upper limit for a dark flow from modeling of the local residual flow of $150 \pm 43 \mathrm{~km} \mathrm{~s}^{-1}$ using SN and galaxy data with a characteristic depth of $z=0.02$. As our highest redshift bin overlaps with the lowest distance bin of the galaxy cluster results of Kashlinsky et al. (2010), we can now perform a model-independent comparison. Our data show no evidence of large bulk flows and are in a $\sim 4 \sigma$ conflict with the claim that the visible Universe is moving as a whole with a velocity of $1000 \mathrm{~km} \mathrm{~s}^{-1}$ relative to the CMB (Kashlinsky et al. 2010). While a recent analyses of the Planck Collaboration (2013) also rejects a dark flow, that study relies on the kSZ effect like Kashlinsky et al. (2010). Our data, on the other hand, allow us to reject a dark flow based on an independent method for the first time. 
The redshift range from 0.03 to 0.08 , for which the SNFACTORY has more than doubled the number of available SNe, bridges the bulk flow measurements obtained from individual galaxy distances to those obtained from galaxy clusters. We have shown in this paper that $\mathrm{SNe}$ are useful to address the outstanding questions about the motion of the $\mathrm{LG}$ relative to the CMB. In this respect our conclusions concerning the role of the SSC should be considered tentative. However, with the ever growing number of observed SNe Ia from active and future surveys, combined with the small systematic uncertainties associated with the measurements, it is only a matter of time before these questions can be settled with certainty.

Acknowledgements. We are grateful to the technical and scientific staff of the University of Hawaii 2.2-m telescope for their assistance in obtaining these data. D. Birchall assisted with acquisition of the data presented here. We also thank the people of Hawaii for access to Mauna Kea. This work was supported in France by CNRS/IN2P3, CNRS/INSU, CNRS/PNC, and used the resources of the IN2P3 computer center. This work was supported by the DFG through TRR33 "The Dark Universe", and by National Natural Science Foundation of China (grant 10903010). U. Feindt acknowledges support by the Bonn-Cologne Graduate School of Physics and Astronomy. C. Wu acknowledges support from the National Natural Science Foundation of China grant 10903010. The IPNL collaborators acknowledge support from the Lyon Institute of Origins under grant ANR-10-LABX-66. This work was also supported by the Director, Office of Science, Office of High Energy and Nuclear Physics and the Office of Advanced Scientific Computing Research, of the U.S. Department of Energy (DOE) under Contract Nos. DE-FG02-92ER40704, DE-AC02-05CH11231, DEFG02-06ER06-04, and DE-AC02-05CH11231; by a grant from the Gordon \& Betty Moore Foundation; by National Science Foundation Grant Nos. AST0407297 (QUEST), and 0087344 \& 0426879 (HPWREN); the France-Berkeley Fund; by an Explora'Doc Grant by the Région Rhône-Alpes.

\section{References}

Aldering, G., Adam, G., Antilogus, P., et al. 2002, in SPIE Conf. Ser. 4836, eds. J. A. Tyson, \& S. Wolff, 61

Aldering, G., Antilogus, P., Bailey, S., et al. 2006, ApJ, 650, 510

Amanullah, R., Stanishev, V., Goobar, A., et al. 2008, A\&A, 486, 375

Amanullah, R., Lidman, C., Rubin, D., et al. 2010, ApJ, 716, 712

Astier, P., Guy, J., Regnault, N., et al. 2006, A\&A, 447, 31

Atrio-Barandela, F. 2013, A\&A, 557, A116

Bailey, S., Aldering, G., Antilogus, P., et al. 2009, A\&A, 500, L17

Barris, B. J., Tonry, J. L., Blondin, S., et al. 2004, ApJ, 602, 571

Bongard, S., Soulez, F., Thiébaut, É., \& Pecontal, É. 2011, MNRAS, 418, 258

Bonvin, C., Durrer, R., \& Kunz, M. 2006, Phys. Rev. Lett., 96, 191302

Branchini, E., Davis, M., \& Nusser, A. 2012, MNRAS, 424, 472

Buton, C., Copin, Y., Aldering, G., et al. 2013, A\&A, 549, A8

Childress, M., Aldering, G., Antilogus, P., et al. 2013a, ApJ, 770, 107

Childress, M., Aldering, G., Antilogus, P., et al. 2013b, ApJ, 770, 108

Chotard, N., Gangler, E., Aldering, G., et al. 2011, A\&A, 529, L4

Colin, J., Mohayaee, R., Sarkar, S., \& Shafieloo, A. 2011, MNRAS, 414, 264

Conley, A., Carlberg, R. G., Guy, J., et al. 2007, ApJ, 664, L13

Courteau, S., Willick, J. A., Strauss, M. A., Schlegel, D., \& Postman, M. 2000, ApJ, 544, 636

Davis, T. M., Hui, L., Frieman, J. A., et al. 2011, ApJ, 741, 67

Djorgovski, S., \& Davis, M. 1987, ApJ, 313, 59

Dressler, A., Lynden-Bell, D., Burstein, D., et al. 1987, ApJ, 313, 42

Erdoğdu, P., Lahav, O., Huchra, J. P., et al. 2006, MNRAS, 373, 45

Gordon, C., Land, K., \& Slosar, A. 2007, Phys. Rev. Lett., 99, 081301

Gordon, C., Land, K., \& Slosar, A. 2008, MNRAS, 387, 371

Grishchuk, L. P., \& Zeldovich, I. B. 1978, Sov. Astron., 2, 125

Guy, J., Astier, P., Baumont, S., et al. 2007, A\&A, 466, 11

Hamuy, M., Phillips, M. M., Suntzeff, N. B., et al. 1996, AJ, 112, 2391

Haugbølle, T., Hannestad, S., Thomsen, B., et al. 2007, ApJ, 661, 650

Hicken, M., Wood-Vasey, W. M., Blondin, S., et al. 2009, ApJ, 700, 1097

Holtzman, J. A., Marriner, J., Kessler, R., et al. 2008, AJ, 136, 2306

Hudson, M. J., Smith, R. J., Lucey, J. R., \& Branchini, E. 2004, MNRAS, 352,

61
Hui, L., \& Greene, P. B. 2006, Phys. Rev. D, 73, 123526

Jha, S., Kirshner, R. P., Challis, P., et al. 2006, AJ, 131, 527

Jha, S., Riess, A. G., \& Kirshner, R. P. 2007, ApJ, 659, 122

Kalus, B., Schwarz, D. J., Seikel, M., \& Wiegand, A. 2013, A\&A, 553, A56

Kashlinsky, A., Atrio-Barandela, F., Kocevski, D., \& Ebeling, H. 2008, ApJ, 686, L49

Kashlinsky, A., Atrio-Barandela, F., Kocevski, D., \& Ebeling, H. 2009, ApJ, 691, 1479

Kashlinsky, A., Atrio-Barandela, F., Ebeling, H., Edge, A., \& Kocevski, D. 2010, ApJ, 712, L81

Kashlinsky, A., Atrio-Barandela, F., \& Ebeling, H. 2011, ApJ, 732, 1

Kashlinsky, A., Atrio-Barandela, F., \& Ebeling, H. 2012 [arXiv: 1202 . 0717]

Keisler, R. 2009, ApJ, 707, L42

Kelly, P. L., Hicken, M., Burke, D. L., Mandel, K. S., \& Kirshner, R. P. 2010, ApJ, 715, 743

Knop, R. A., Aldering, G., Amanullah, R., et al. 2003, ApJ, 598, 102

Kocevski, D. D., \& Ebeling, H. 2006, ApJ, 645, 1043

Kocevski, D. D., Ebeling, H., Mullis, C. R., \& Tully, R. B. 2007, ApJ, 662, 224

Kogut, A., Lineweaver, C., Smoot, G. F., et al. 1993, ApJ, 419, 1

Kowalski, M., Rubin, D., Aldering, G., et al. 2008, ApJ, 686, 749

Krisciunas, K., Garnavich, P. M., Challis, P., et al. 2005, AJ, 130, 2453

Lampeitl, H., Smith, M., Nichol, R. C., et al. 2010, ApJ, 722, 566

Lavaux, G., \& Hudson, M. J. 2011, MNRAS, 416, 2840

Lavaux, G., Tully, R. B., Mohayaee, R., \& Colombi, S. 2010, ApJ, 709, 483

Lavaux, G., Afshordi, N., \& Hudson, M. J. 2013, MNRAS, 430, 1617

Lim, S., \& Lee, J. 2013, JCAP, 01, 019

Lynden-Bell, D., Faber, S. M., Burstein, D., et al. 1988, ApJ, 326, 19

Ma, Y.-Z., \& Scott, D. 2013, MNRAS, 428, 2017

Macaulay, E., Feldman, H., Ferreira, P. G., Hudson, M. J., \& Watkins, R. 2011, MNRAS, 414, 621

Macaulay, E., Feldman, H. A., Ferreira, P. G., et al. 2012, MNRAS, 425, 1709

Miknaitis, G., Pignata, G., Rest, A., et al. 2007, ApJ, 666, 674

Mody, K., \& Hajian, A. 2012, ApJ, 758, 4

Muñoz, J. A., \& Loeb, A. 2008, MNRAS, 391, 1341

Nusser, A., \& Davis, M. 2011, ApJ, 736, 93

Nusser, A., Branchini, E., \& Davis, M. 2011, ApJ, 735, 77

Osborne, S. J., Mak, D. S. Y., Church, S. E., \& Pierpaoli, E. 2011, ApJ, 737, 98

Peebles, P. J. E. 1993, Principles of physical cosmology, Princeton series in physics edn. (Princeton University Press)

Perlmutter, S., Aldering, G., Goldhaber, G., et al. 1999, ApJ, 517, 565

Phillips, M. M. 1993, ApJ, 413, L105

Planck Collaboration 2013, A\&A, submitted [arXiv: 1303 . 5090]

Raychaudhury, S., Fabian, A. C., Edge, A. C., Jones, C., \& Forman, W. 1991, MNRAS, 248, 101

Regnault, N., Conley, A., Guy, J., et al. 2009, A\&A, 506, 999

Riess, A. G., Press, W. H., \& Kirshner, R. P. 1995, ApJ, 445, L91

Riess, A. G., Filippenko, A. V., Challis, P., et al. 1998, AJ, 116, 1009

Riess, A. G., Kirshner, R. P., Schmidt, B. P., et al. 1999, AJ, 117, 707

Riess, A. G., Strolger, L.-G., Casertano, S., et al. 2007, ApJ, 659, 98

Scalzo, R., Aldering, G., Antilogus, P., et al. 2012, ApJ, 757, 12

Scalzo, R. A., Aldering, G., Antilogus, P., et al. 2010, ApJ, 713, 1073

Scaramella, R. 1995, Astrophys. Lett. Commun., 32, 137

Scaramella, R., Baiesi-Pillastrini, G., Chincarini, G., Vettolani, G., \& Zamorani, G. 1989, Nature, 338, 562

Schlegel, D. J., Finkbeiner, D. P., \& Davis, M. 1998, ApJ, 500, 525

Schwarz, D. J., \& Weinhorst, B. 2007, A\&A, 474, 717

Shapley, H. 1930, Harvard College Observatory Bulletin, 874, 9

Sheth, R. K., \& Diaferio, A. 2011, MNRAS, 417, 2938

Sullivan, M., Conley, A., Howell, D. A., et al. 2010, MNRAS, 406, 782

Tonry, J., \& Schneider, D. P. 1988, AJ, 96, 807

Tonry, J. L., Schmidt, B. P., Barris, B., et al. 2003, ApJ, 594, 1

Tripp, R. 1998, A\&A, 331, 815

Tully, R. B., \& Fisher, J. R. 1977, A\&A, 54, 661

Tully, R. B., Shaya, E. J., Karachentsev, I. D., et al. 2008, ApJ, 676, 184

Turnbull, S. J., Hudson, M. J., Feldman, H. A., et al. 2012, MNRAS, 420, 447

Turner, M. S. 1991, Phys. Rev. D, 44, 3737

Watkins, R., Feldman, H. A., \& Hudson, M. J. 2009, MNRAS, 392, 743

Weyant, A., Wood-Vasey, M., Wasserman, L., \& Freeman, P. 2011, ApJ, 732, 65

Wiltshire, D. L., Smale, P. R., Mattsson, T., \& Watkins, R. 2013, Phys. Rev. D, 88,083529 